\title{
Cardiac Scintigraphy with Bone Markers in Clinical Practice: When to Solicit the Exam? How to Interpret the Results?
}

\author{
Marcus Vinicius Simões ${ }^{(0)}$ \\ Faculdade de Medicina de Ribeirão Preto da Universidade de São Paulo, Ribeirão Preto, SP - Brazil
}

One of the greatest and most recent advances in clinical management of cardiac amyloidosis (CA), which has completely reshaped clinical practice, took place in the field of diagnosis, based on the possibility of using molecular scintigraphy images for non-invasive detection of amyloid deposits in the myocardium, making endomyocardial biopsy unnecessary to conclude diagnosis. ${ }^{1}$

There are some fundamental aspects that clinicians need to know in order to apply this powerful tool in their practice, choosing wisely the best time to request it and how to interpret the results.

Preliminarily, it is important for us to review the main studies that have offered signposts for the application of scintigraphy with bone markers for diagnosis of CA.

In one of the first studies to demonstrate this application, Wizenberg et al. evaluated 10 consecutive patients with biopsy-confirmed CA, having observed that all patients exhibited intense and diffuse anomalous myocardial uptake on cardiac scintigraphy with ${ }^{99 m}{ }^{T c}$-pyrophosphate. ${ }^{2}$ However, later studies showed variable yield of these images in patients with different forms of $\mathrm{CA}$, underscoring $\mathrm{CA}$ associated with immunoglobulin light chains (AL-CA), in addition to transthyretin-associated CA (ATTR-CA). The mechanisms by which bone radiotracers are accumulated in tissue deposits of amyloid protein probably involve the presence of microcalcifications in these protein aggregates, as these radiotracers are highly avid for calcium deposits. It is this aspect that also makes it possible for us to detect accumulation of these radiotracers in the hearts of patients with ischemic heart disease with recent acute myocardial infarction, and this was, historically, the first clinical application of this type of image.

The progress of knowledge with respect to the clinical applicability of cardiac scintigraphy images with bone radiotracers for diagnosis of CA occurred with the seminal study by Enrica Perugini et al., who investigated 10 patients with ATTR-CA and 15 patients with AL-CA, in addition to 10 control patients who were not affected by CA. ${ }^{3}$ In this study, by means of visual analysis of planar chest images, complemented by analysis of SPECT images, all patients with

\section{Keywords}

Amyloidosis; Scintigraphy; Diagnostic Methods.

Mailing Address: Marcus Vinicius Simões •

Av. Bandeirantes 3.900 - Campus da USP - Bairro Monte Alegre, Postal Code: 14049-900

E-mail: msimoes@fmrp.usp.br

Manuscript received September 12, 2021, revised manuscript September 24, 2021, accepted September 24, 2021

DOI: https://doi.org/10.36660/abchf.20210022
ATTR-CA exhibited intense anomalous radiotracer uptake in the myocardium at a comparative intensity equivalent to (grade 2) or higher than (grade 3) that seen in the costal arches. In contrast, all patients with AL-CA and all controls exhibited absence of cardiac uptake (grade 0). Thus, visual analysis of Perugini cardiac uptake grade was shown to be useful for differentiating between the main forms of CA (ATTR and AL), suggesting that positive myocardial uptake could indicate the presence of ATTR-CA.

Nevertheless, it was later identified that a significant proportion, approximately $20 \%$, of patients with AL-CA could also exhibit grade 2 or 3 of cardiac uptake of bone markers, making it essential to rule out the presence of immunoglobulin light chains in order to confirm diagnosis of ATTR-CA in patients with positive cardiac scintigraphy using bone markers. ${ }^{4}$

In another fundamental study, Gillmore et al. tested the diagnostic application of scintigraphy with bone markers, compared to the result of endomyocardial biopsy, in an expressive sample of 374 patients, seeking to identify those with ATTR-CA. ${ }^{5}$ The general results showed a high sensitivity for scintigraphy, above $99 \%$, but a reduced specificity of $86 \%$, for diagnosis of ATTR-CA. The false positives observed in this study were almost entirely due to the presence of uptake in patients with AL-CA. Thus, analysis of the results based on the double criteria (scintigraphy with grade 2 or 3 uptake, in addition to the absence of light chains), obtained 100\% specificity and predictive value, thus defining the non-invasive criteria for diagnosis of ATTR-CA, which have gone on to become a standard of conduct worldwide (Figure 1). ${ }^{5}$ Sensitivity for diagnosing ATTR-CA, according to these criteria, was 70\%. ${ }^{5}$

\section{When to solicit cardiac scintigraphy with bone markers?}

In view of the aspects mentioned above, it is important for investigation of the specific form of ATTR-CA, using bone marker scintigraphy, to begin after 2 key preliminary steps: 1 . establishing high clinical suspicion of the presence of CA and 2. ruling out the presence of immunoglobulin light chains, which would lead us to diagnosis of AL-CA.

It is fundamental to establish high clinical suspicion, given that negative scintigraphy does not rule out the presence of CA in its various forms, particularly the AL form. In other words, in a significant percentage of cases with high clinical suspicion and negative scintigraphy, investigation should continue with tissue biopsy, including endomyocardial biopsy, in order to conclude diagnosis. It is clear that endomyocardial biopsy should only be performed in cases of high clinical suspicion, as it is an invasive method with risks that, although low, are inherent to the procedure.

On the other hand, it is fundamental to rule out the presence of light chains previously, so that a positive scintigraphy result is not taken as evidence of ATTR-CA. This 

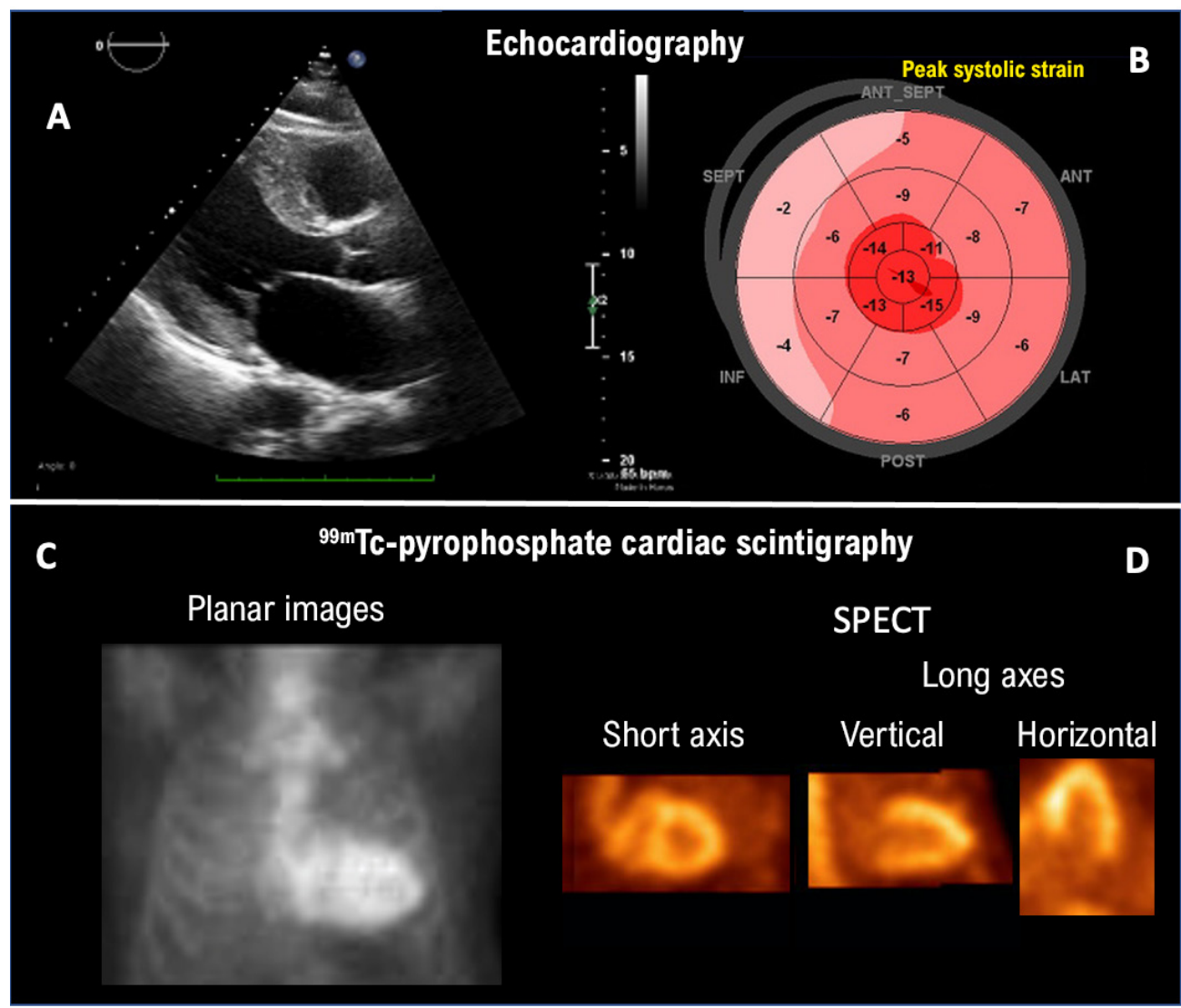

Figure 1 - Illustrative images of a 73-year-old male patient with symptoms of heart failure with preserved ejection fraction, showing changes compatible with ATTR-CA, after laboratory tests showing absence of immunoglobulin light chains: (A) structural alterations on echocardiogram and increased left ventricular wall thickness (interventricular septum $=14 \mathrm{~mm}$ ); $(B)$ images of global longitudinal strain on a polar map showing the sign of apical sparing; (C) planar 99mTc-pyrophosphate cardiac scintigraphy images, showing intense radiotracer uptake over the heart (Perugini grade 3, greater than that of the costal arches), which were confirmed in the myocardial walls of the left and right ventricles on SPECT images. (D) Genetic analysis was negative for transthyretin gene mutations, characterizing diagnosis of wild type ATTR-CA.

misdiagnosis could delay or exclude patients with AL-CA from chemotherapy/bone marrow transplantation, which could save the patient's life. On the other hand, it is worth remembering that $\mathrm{AL}-\mathrm{CA}$ has very poor prognosis, with a median survival of 6 to 8 months in cases with heart failure and late diagnosis, which highlights the urgent need to rule out this diagnosis during the initial moments of investigation of suspected cases of CA.

The hypothesis that scintigraphy with bone markers could be used as a screening tool in elderly men with heart failure with preserved ejection fraction who exhibit the warning signs for CA has been tested in several clinical studies, but the value of this strategy has yet to be defined. ${ }^{6}$

\section{How to interpret the results?}

When interpreting cardiac scintigraphy images with bone markers in patients with suspected ATTR-CA, it is important that we keep 2 essential aspects in mind:
1. Positive images for ATTR-CA should have triple confirmation: 1. Perugini grade 2 or 3 cardiac uptake, 2. heart to contralateral ratio $>1.5$ on imaging 1 hour after radiotracer injection, and 3 . confirmation of radiopharmaceutical concentration in the ventricular myocardium in the SPECT images, as illustrated in Figure 1.

2. There are common conditions in clinical practice that lead to false positive cases for ATTR-CA, including acute myocardial infarction, $\mathrm{AL}-\mathrm{CA}$, and uptake restricted to the blood pool in planar images, which can be misinterpreted as a positive exam.

The most frequent cause of diagnostic error, with "false positive" scintigraphy exam for ATTR-CA, is AL-CA. In fact, these cases are "scintigraphically positive," but they should be interpreted in light of the knowledge that $20 \%$ to $30 \%$ of patients with AL-CA may have grade 2 or 3 positive scintigraphy. In this manner, preliminary exclusion of the presence of light chains is fundamental in order to avoid this "false positive." 


\section{Viewpoint}

Another false positive, which results from the interpretation of planar scintigraphy images, without confirmation from SPECT images, is increased uptake in the cardiac area in planar images associated with radioactivity present in the blood pool, not located in the myocardium. ${ }^{1}$ If we consider that the application of this imaging method for this purpose is still recent in Brazil, a careful review of positive tests performed by the nuclear physician together with the requesting clinician is desirable, in order to safely rule out this type of false positive test, not losing sight of the fundamental importance of these exam results to non-invasive diagnosis of ATTR-CA.

It is worth remembering here that ischemic heart disease, corresponding to cases with more severe myocardial ischemia, acute myocardial infarction, or recurrent ischemia at rest, may exhibit significant accumulation of the bone radiotracer. It is, therefore, essential to rule out severe coronary artery disease in patients with risk factors, ischemic changes on electrocardiogram, or angina pectoris, as an essential initial diagnostic step in these cases.

Finally, it is necessary to remember that there are false negatives of bone marker scintigraphy in patients with confirmed ATTR-CA. This can occur in patients with hereditary ATTR with mutations that are known not to capture bone markers, such as early-onset V50M and P64L. Accordingly, cases with high clinical suspicion of $\mathrm{CA}$ in which the presence of light chains has been ruled out and which exhibit negative bone marker scintigraphy, may proceed to endomyocardial biopsy to confirm/reject diagnosis of the disease.

\section{Author contributions}

Conception and design of the research: Simões MV. Acquisition of data: Simões MV. Analysis and interpretation of the data: Simões MV. Statistical analysis: Simões MV. Obtaining financing: Simões MV. Writing of the manuscript: Simões MV. Critical revision of the manuscript for intellectual content: Simões MV. Supervision / as the major investigador: Simões MV.

\section{Potential Conflict of Interest}

No potential conflict of interest relevant to this article was reported.

\section{Sources of Funding}

There were no external funding sources for this study.

\section{Study Association}

This study is not associated with any thesis or dissertation work.

\section{Ethics approval and consent to participate}

This article does not contain any studies with human participants or animals performed by any of the authors.

\section{References}

1. Hanna M, Ruberg FL, Maurer MS, Dispenzieri A, Dorbala S, Falk RH, et al. Cardiac Scintigraphy with Technetium-99m-Labeled Bone-Seeking Tracers for Suspected Amyloidosis: JACC Review Topic of the Week. J Am Coll Cardiol. 2020;75(22):2851-62. doi: 10.1016/j.jacc.2020.04.022.

2. Wizenberg TA, Muz J, Sohn YH, Samlowski W, Weissler AM. Value of Positive Myocardial Technetium-99m-Pyrophosphate Scintigraphy in the Noninvasive Diagnosis of Cardiac Amyloidosis. Am Heart J. 1982;103(4):468-73. doi: 10.1016/0002-8703(82)90331-3.

3. Perugini E, Guidalotti PL, Salvi F, Cooke RM, Pettinato C, Riva L, et al. Noninvasive Etiologic Diagnosis of Cardiac Amyloidosis Using 99mTc3,3-Diphosphono-1,2-Propanodicarboxylic Acid Scintigraphy. J Am Coll Cardiol. 2005;46(6):1076-84. doi: 10.1016/j.jacc.2005.05.073.

4. Quarta CC, Zheng J, Hutt D, Grigore SF, Manwani R, Sachchithanantham S, et al. 99mTc-DPD Scintigraphy in Immunoglobulin Light Chain (AL) Cardiac Amyloidosis. Eur Heart J Cardiovasc Imaging. 2021:jeab095. doi: 10.1093/ ehjci/jeab095.

5. Gillmore JD, Maurer MS, Falk RH, Merlini G, Damy T, Dispenzieri A, et al. Nonbiopsy Diagnosis of Cardiac Transthyretin Amyloidosis. Circulation. 2016;133(24):2404-12. doi: 10.1161/CIRCULATIONAHA.116.021612.

6. González-López E, Gallego-Delgado M, Guzzo-Merello G, Haro-Del Moral FJ, Cobo-Marcos M, Robles C, et al. Wild-Type Transthyretin Amyloidosis as a Cause of Heart Failure with Preserved Ejection Fraction. Eur Heart J. 2015;36(38):2585-94. doi: 10.1093/eurheartj/ehv338. 